\title{
MARS14 Simulation of Fermilab 120-GeV Beam Dumps
}

\author{
N.V. Mokhov \\ Fermi National Accelerator Laboratory \\ P.O. Box 500, Batavia, Illinois 60510
}

March 28, 2003

To estimate groundwater activation around the Fermilab Switchyard, Meson Target Train and M03 Meson beam dumps at $120 \mathrm{GeV}$, express MARS14 [1] simulations have been performed. A proton beam RMS spot size is $\sigma_{x}=\sigma_{y}=1 \mathrm{~cm}$ in the first two cases, while $\sigma_{x}=\sigma_{y}=2 \mathrm{~cm}$ for M03. Calculated are star density distributions (above $50 \mathrm{MeV}$ ) normalized per one incident proton. The configurations proposed by Chuck Brown have been implemented into the MARS code.

Switchyard Dump. Its geometry as implemented into the code is shown in Fig. 1. Densities of the materials used (in $\mathrm{g} / \mathrm{cm}^{3}$ ) are 2.7 (aluminum), 7.2 (iron), 2.35 (ordinary concrete) and 2.24 (Fermilab wet dirt). Fig. 2 shows two-dimensional star density isocontours. Radial distribution at shower maximum and longitudinal distribution along z-axis are shown in Fig. 3.

Meson Target Train Dump. Its geometry as implemented into the MARS code is shown in Figs. 4 and 5. Densities of the materials used (in $\mathrm{g} / \mathrm{cm}^{3}$ ) are 8.96 (copper), 7.2 (iron) and 2.2 (sand+gravel). Fig. 6 shows two-dimensional azimuthally averaged star density isocontours, while Fig. 7 presents star density isocontours in a transverse slice at $50<\mathrm{z}<100 \mathrm{~cm}$. Lateral distribution at shower maximum and longitudinal distribution along z-axis are shown in Fig. 8.

M03 Dump. Its geometry as implemented into the code is shown in Figs. 9 and 10 along with particle tracks for several protons hitting the dump. Densities of the materials used (in $\mathrm{g} / \mathrm{cm}^{3}$ ) are 7.2 (iron), 0.00121 (air), 2.35 (ordinary concrete) and 2.24 (Fermilab wet dirt). Fig. 11 shows two-dimensional azimuthally averaged star density isocontours, while Fig. 12 presents star density isocontours in a transverse slice at $100<\mathrm{z}<150 \mathrm{~cm}$. Horizontal and vertical distributions at maximum are shown in Fig. 13. Fig. 14 presents longitudinal distribution along z-axis.

\section{References}

[1] N.V. Mokhov, "The MARS Code System User's Guide", Fermilab-FN-628 (1995); N.V. Mokhov, O.E. Krivosheev, "MARS Code Status", Proc. Monte Carlo 2000 Conf., p. 943, Lisbon, October 23-26, 2000; Fermilab-Conf-00/181 (2000); http://www-ap.fnal.gov/MARS/. 


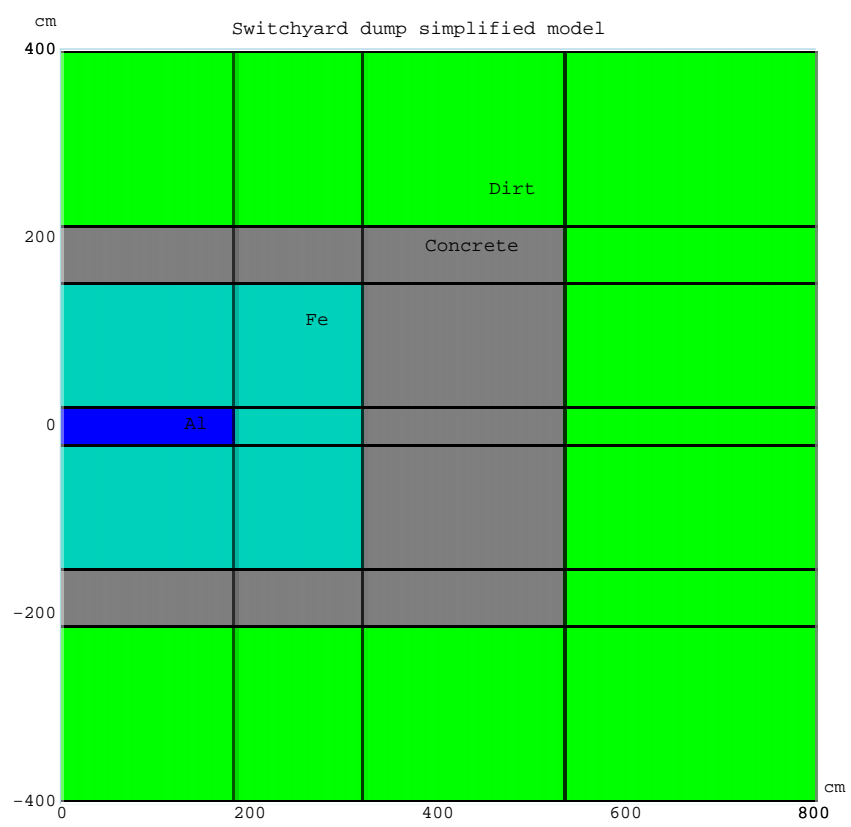

$\stackrel{x}{\rightarrow}$

Figure 1: Switchyard dump MARS model.

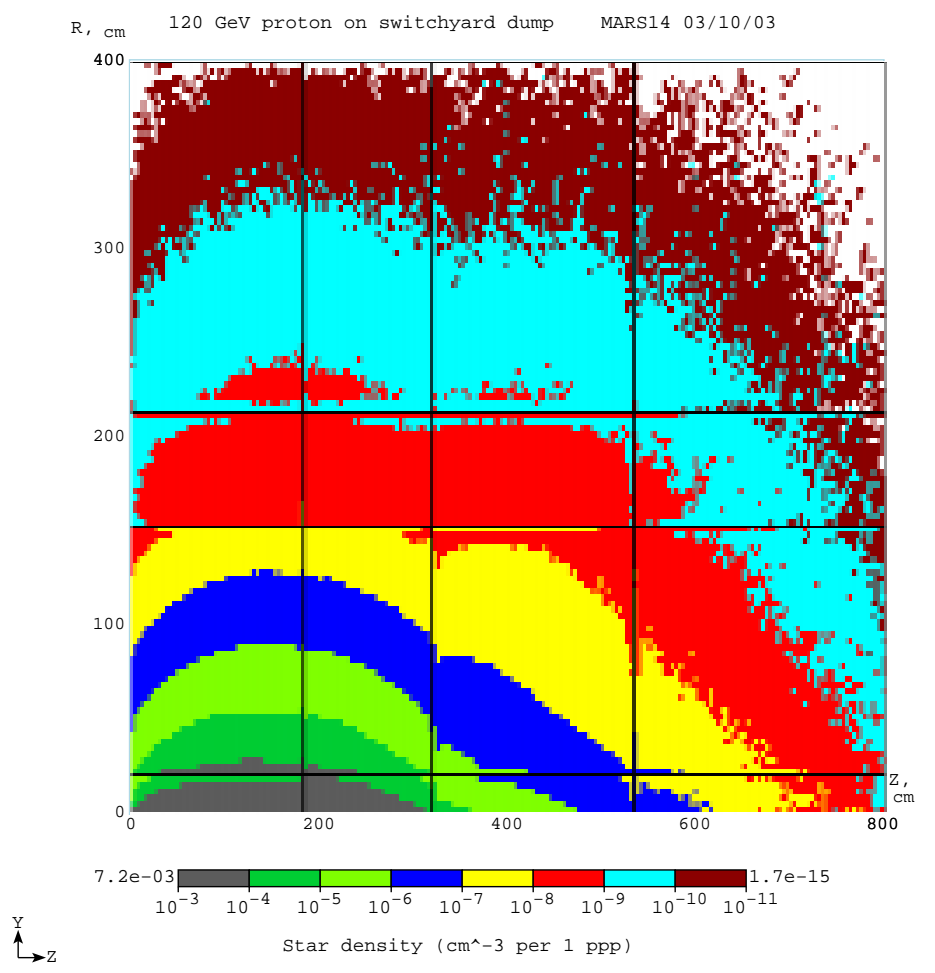

Figure 2: Star density isocontours in Switchyard dump. 

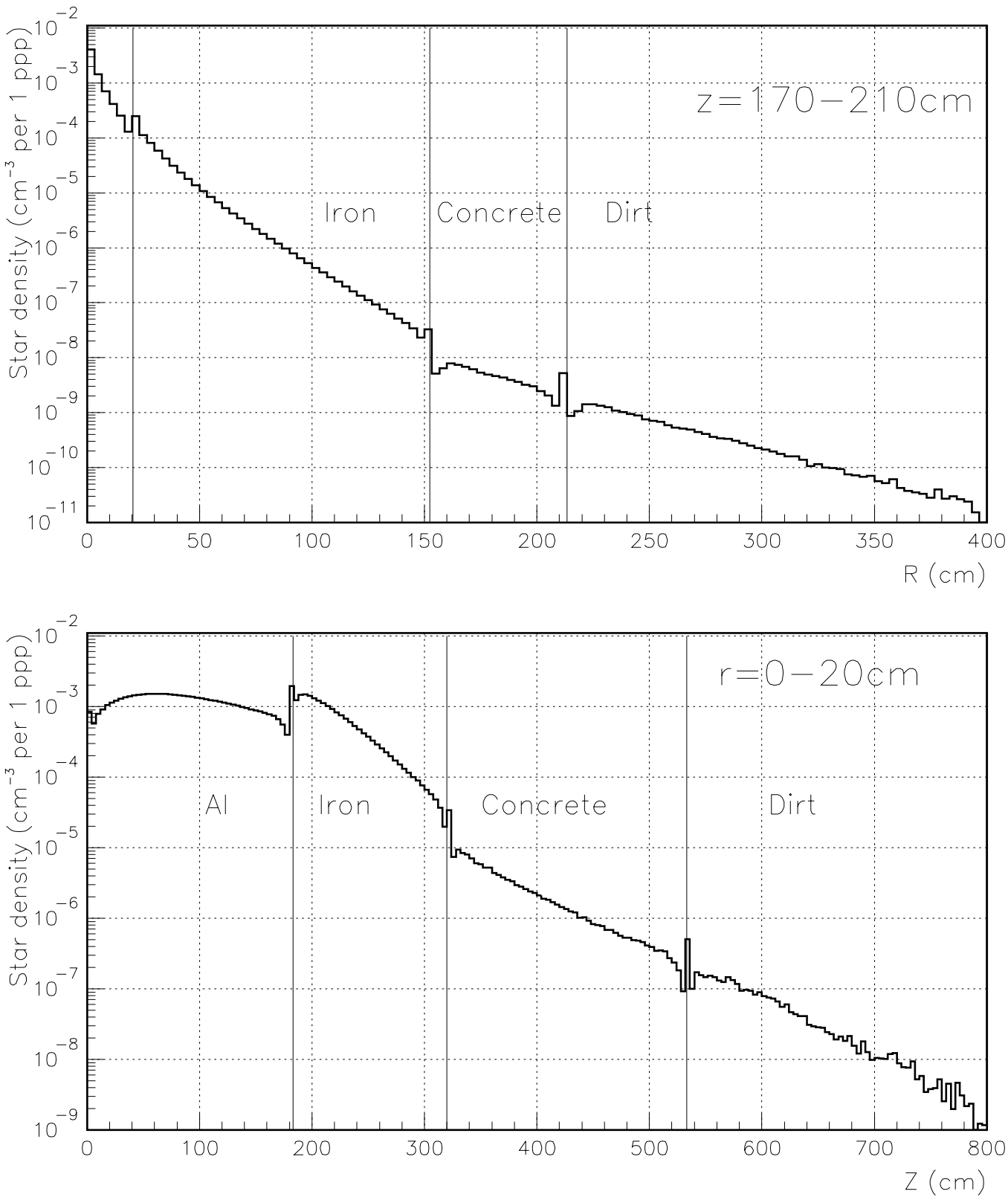

Figure 3: Radial (top) and longitudinal (bottom) distributions of maximum star density in Switchyard dump. 


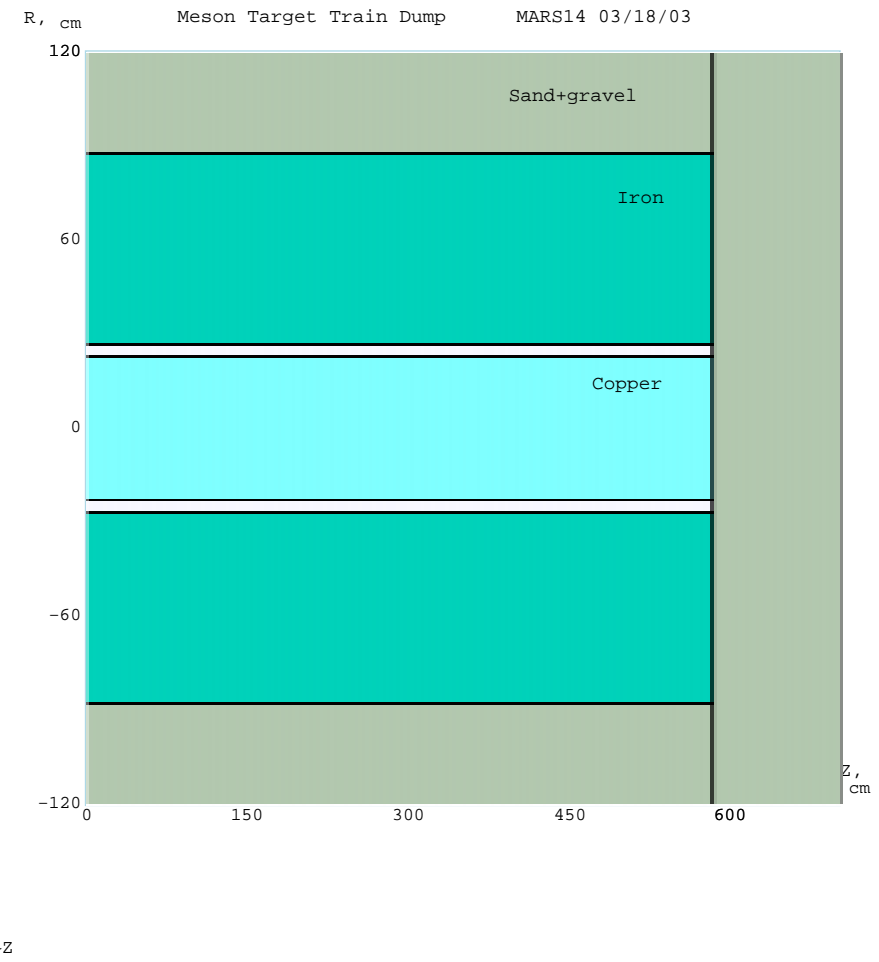

Figure 4: Meson Target Train dump MARS model: longitudinal view.

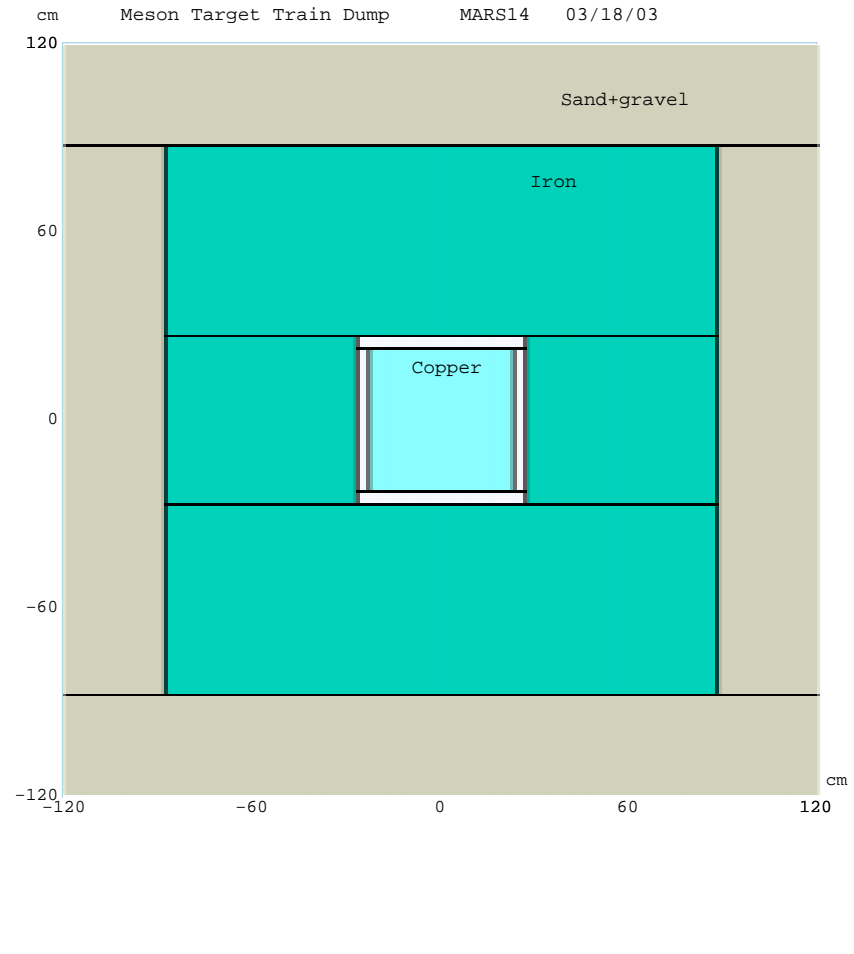

Figure 5: Meson Target Train dump MARS model: lateral view. 


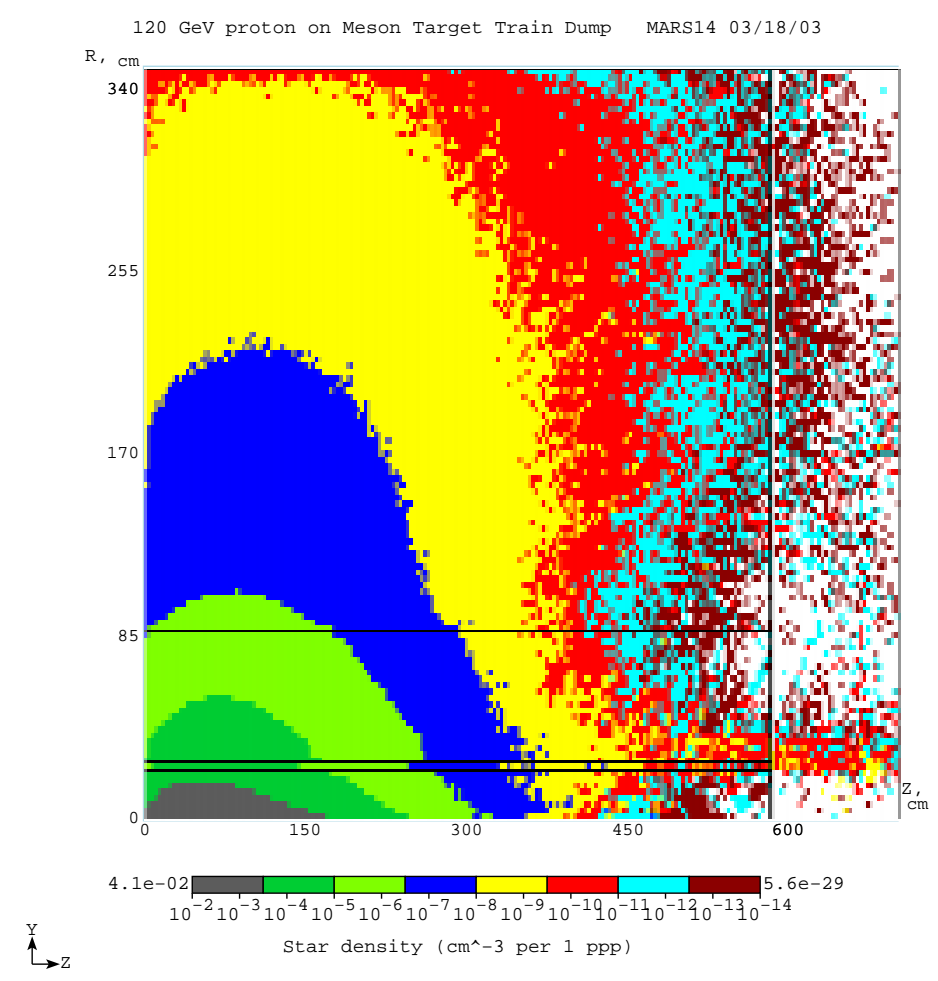

Figure 6: Azimuthally averaged star density isocontours in Meson Target Train dump.

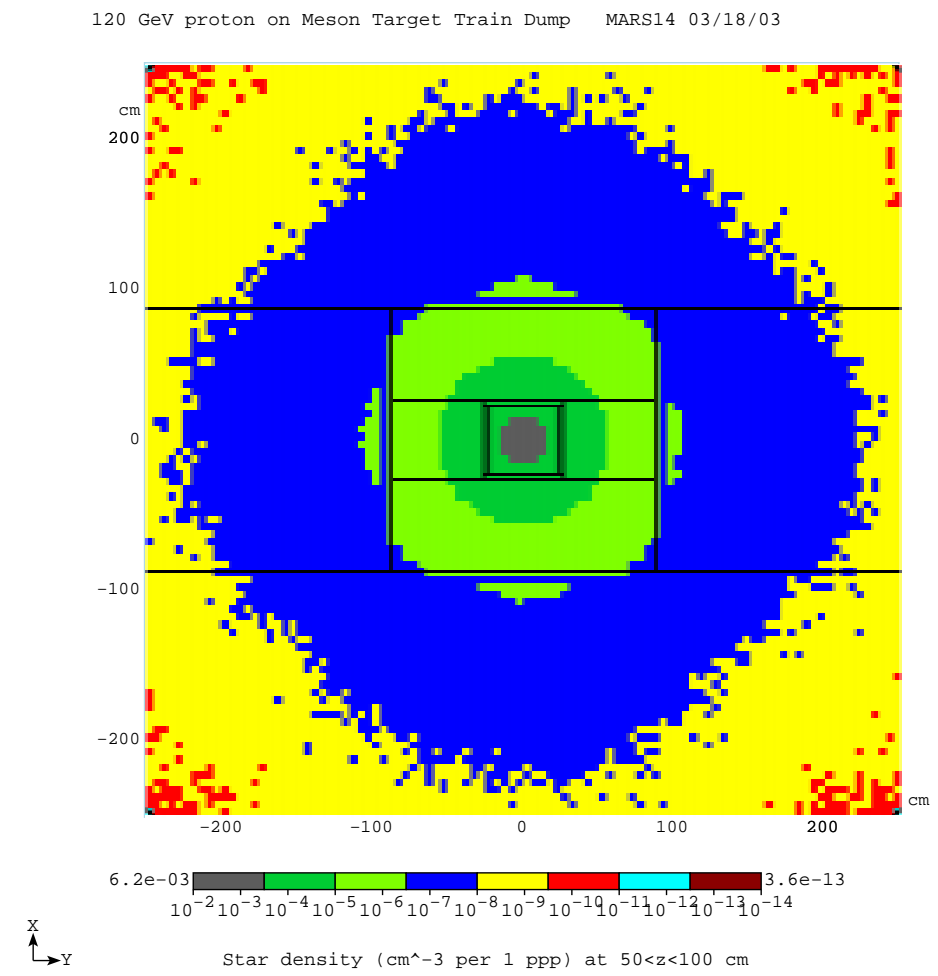

Figure 7: Star density isocontours in a transverse slice at $50<\mathrm{z}<100 \mathrm{~cm}$ in Meson Target Train dump. 

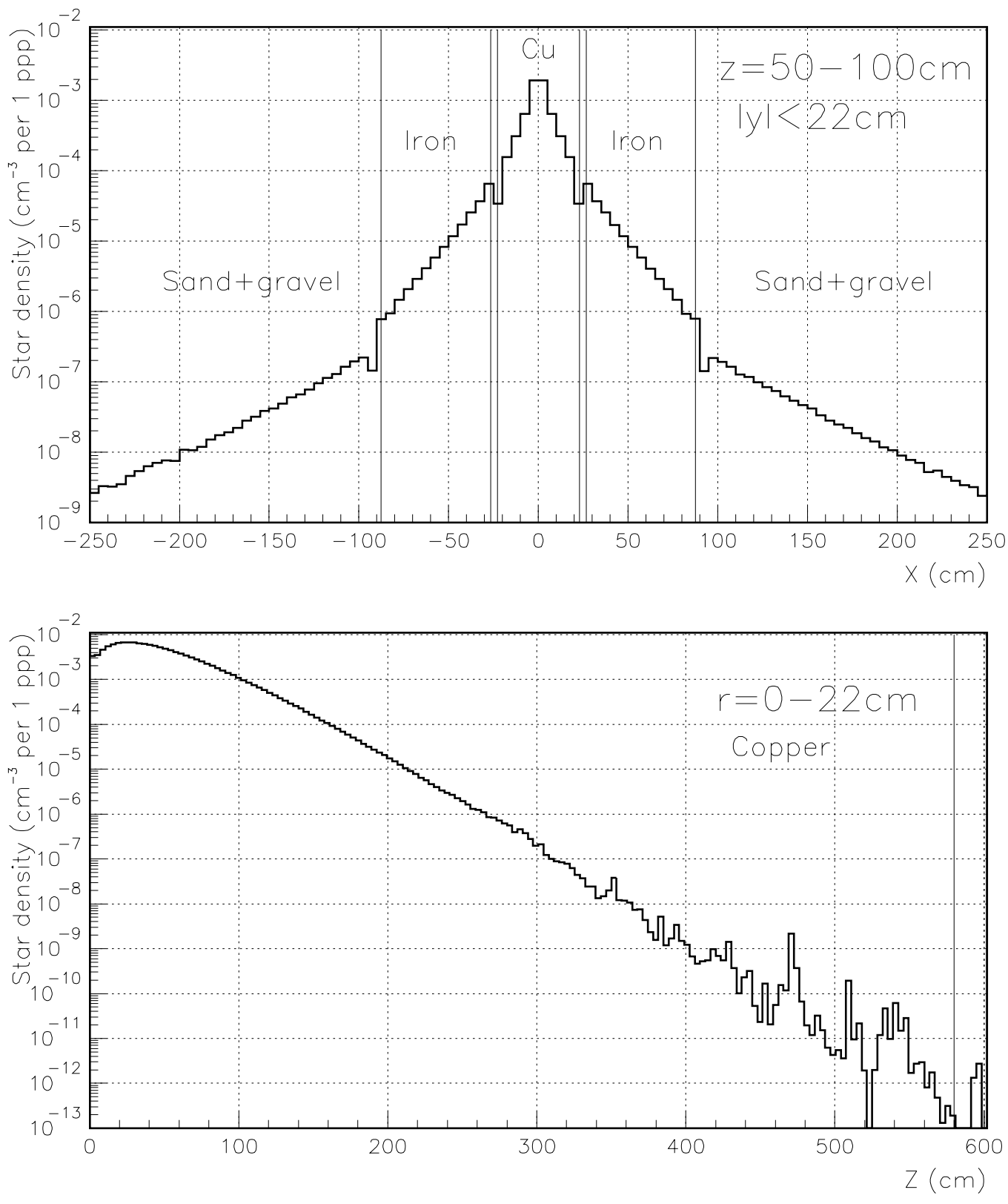

Figure 8: Lateral (top) and longitudinal (bottom) distributions of maximum star density in Meson Target Train dump. 




Figure 9: M03 beam dump setup MARS model: longitudinal view with particle tracks generated by several $120-\mathrm{GeV}$ protons hitting the dump.

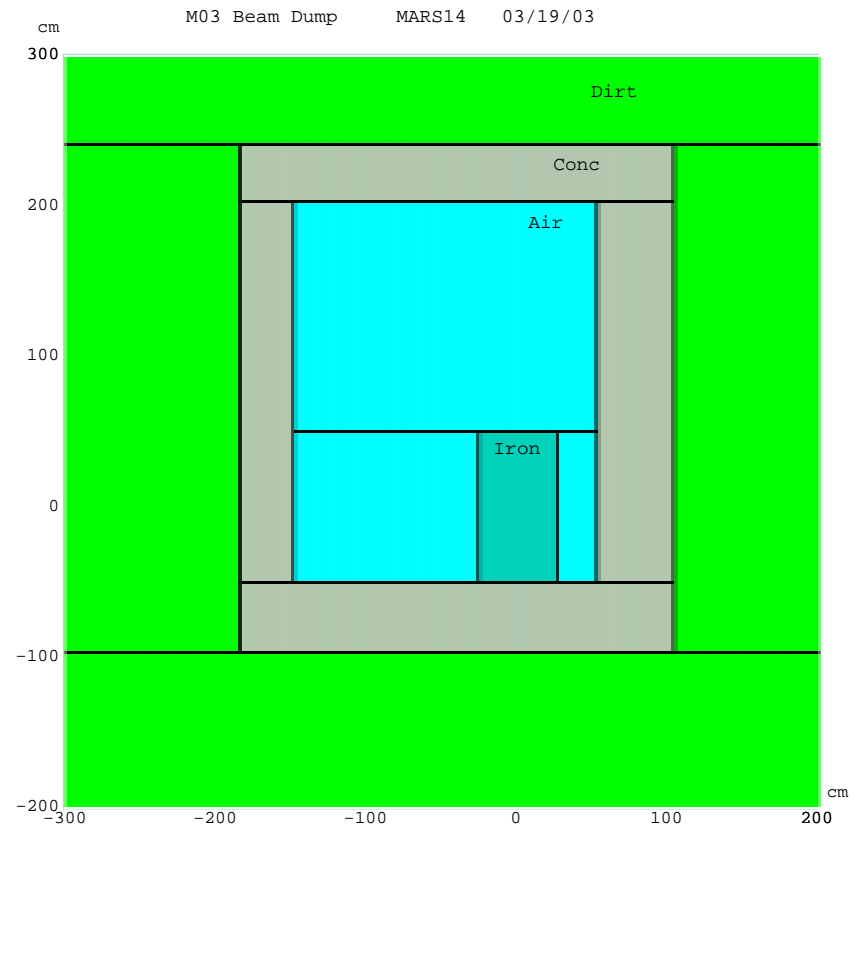

Figure 10: M03 beam dump setup MARS model: lateral view. 


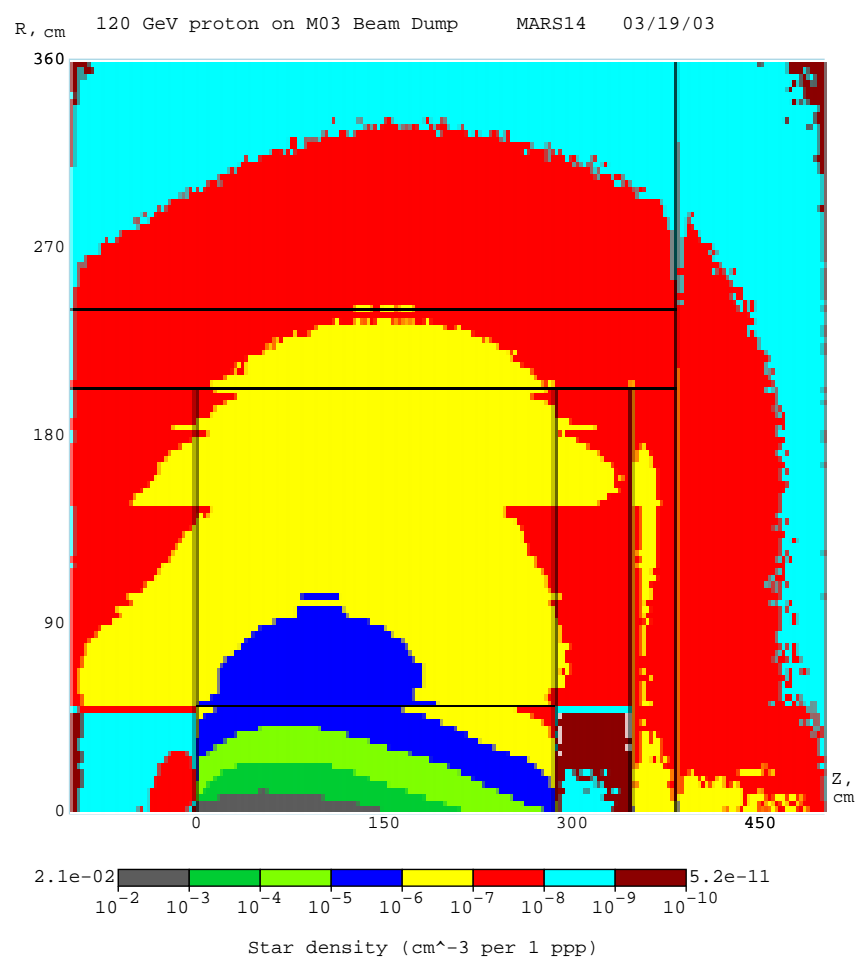

Figure 11: Azimuthally averaged star density isocontours in the M03 beam dump setup.

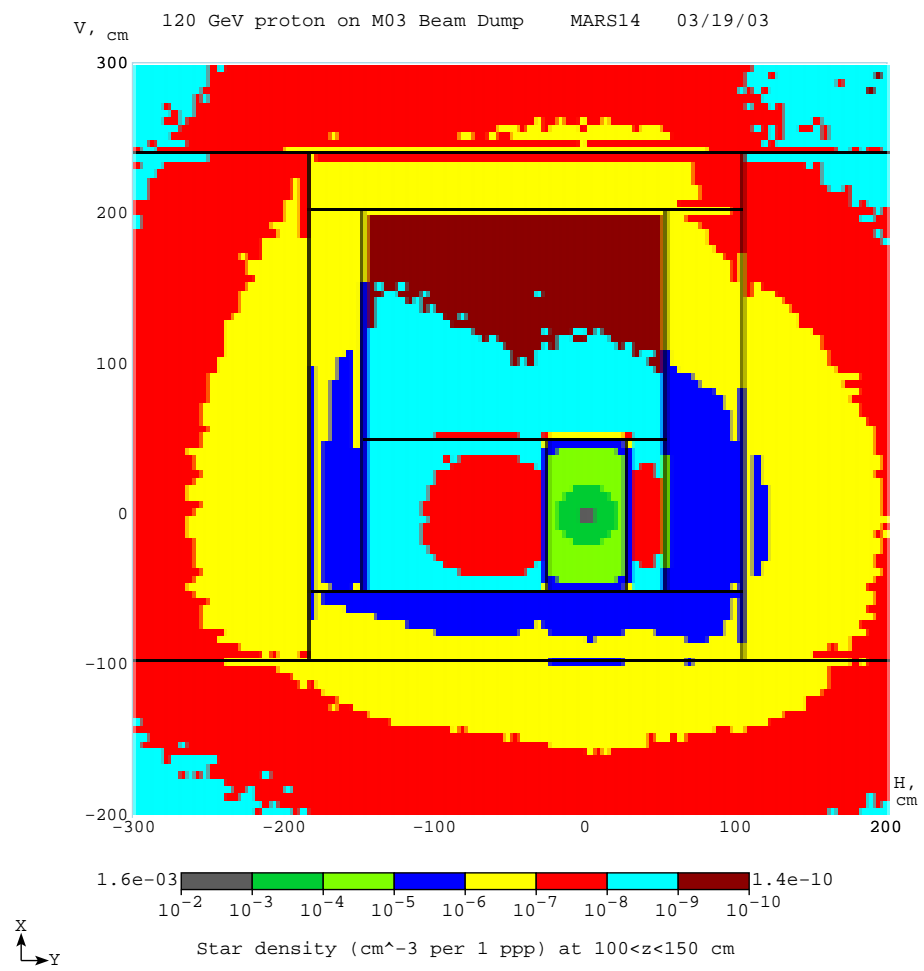

Figure 12: Star density isocontours in a transverse slice at $100<\mathrm{z}<150 \mathrm{~cm}$ in the M03 beam dump setup. 

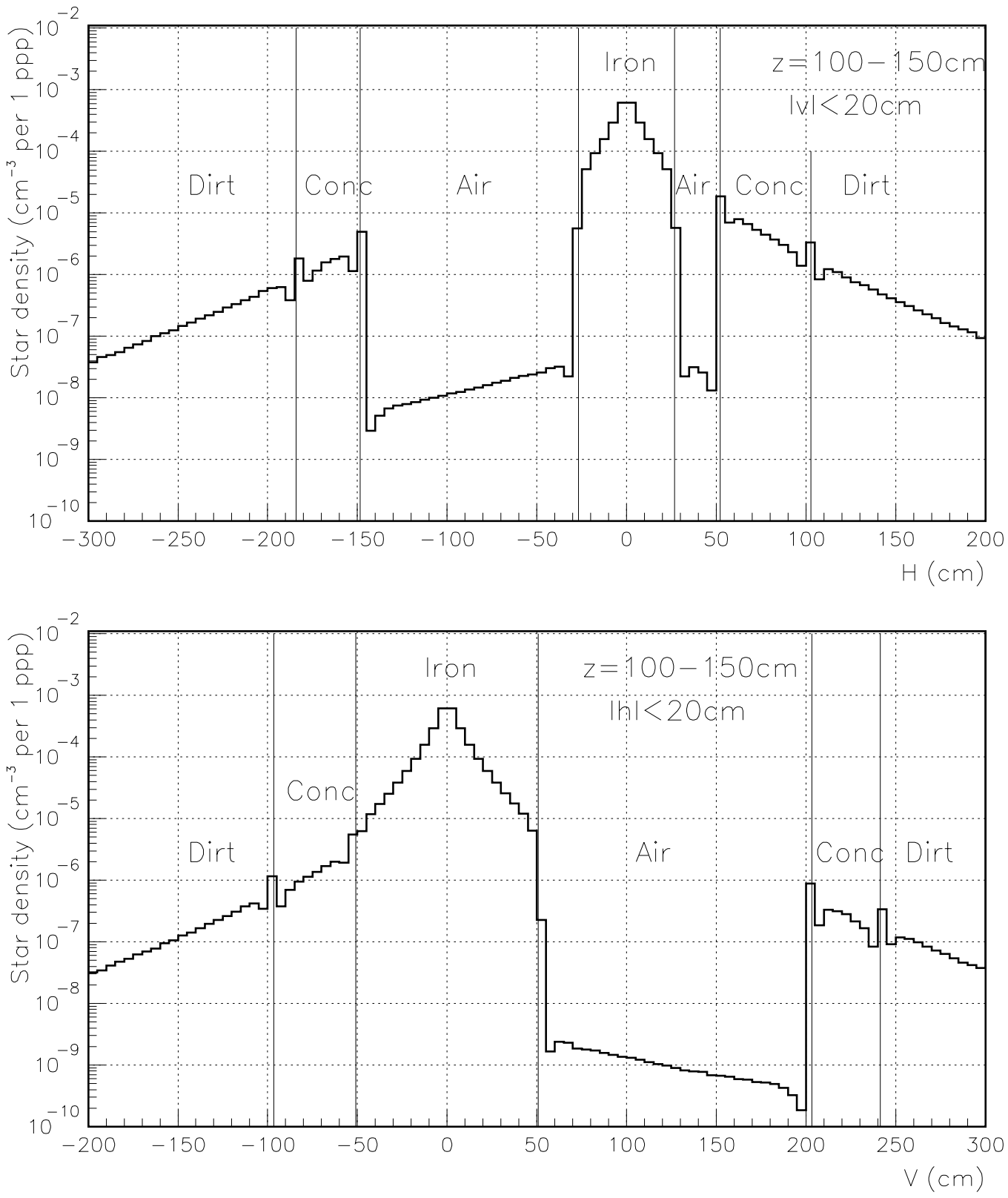

Figure 13: Horizontal (top) and vertical (bottom) distributions of star density in a \pm 20 -cm band of the M03 beam dump setup at maximum $(100<\mathrm{z}<150 \mathrm{~cm})$. 


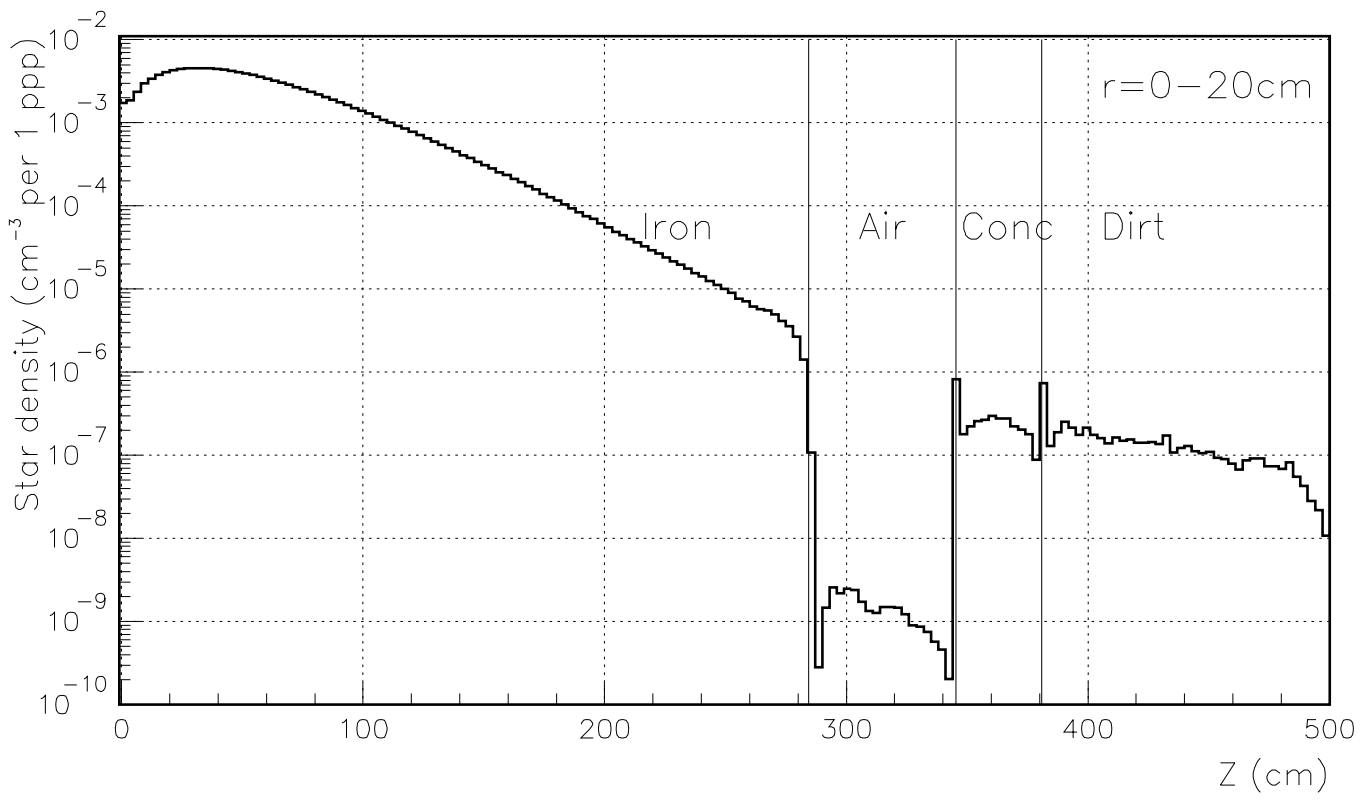

Figure 14: Longitudinal distribution of star density along the axis of the M03 beam dump setup. 